\section{The Commercial Development of FT-IR Microspectroscopy}

John A. Reffner, Ph.D.

Spectra-Tech, Inc., Stamford, CT 06906

Pittcon '93 marked the 10th anniversary of the introduction of a microscope attachment designed specifically for Fourier transform infrared microspectroscopy. The first commercial microscope developed for FT-IR spectroscopy was designed by Spectra-Tech, Inc., under the direction of then-owner D. W. Sting, in fulfillment of a contract with C. T. Foskett of the Digilab Division of BioRad. Digilab recognized a growing interest in the market for an FT-IR microscope and contracted with Spectra-Tech to design and build a microscope accessory for Digilab's FT-IR spectrometers. This microscope was introduced at the 1983 Pittsburgh Conference.

Linking microscopy with Fourier transform spectoscopy was a very significant event, but the foundation of infrared microspectroscopy can be traced back to 1949 . The explosive growth in the use of infrared-absorption spectroscopy following World War II led researchers R. Gore (in the U.S.A.), and R. Barer, A. R. Cole, and H. W. Thompson (in England), to investigate (in 1949) the possibility of recording infrared spectra of microscopic samples. Their instrumental set-ups were similar, consisting of a high intensity source, a microscope with an all-reflecting condenser and matched objective, and a dispersive single-beam infrared spectometer (see Fig. 1). All-reflecting microscopes were developed by C. R. Burch (in 1947) and D. S. Grey (in 1949) to improve spatial resolution in ultraviolet microscopy. The advantage of all-reflecting microscopes was their freedom from both chromatic aberration and absorption by refracting lenses. These first microscopes formed the basis for all subsequent optical designs.

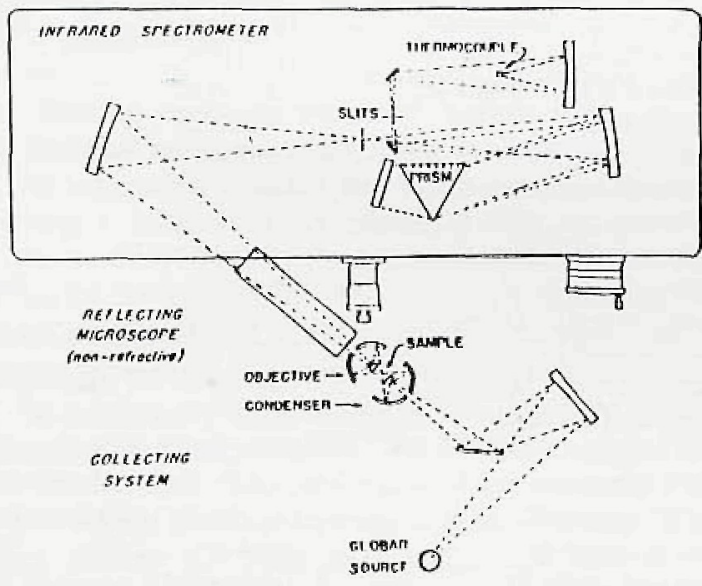

Figure 1: Diagram of an infrared microspectrometer from "Infrared Spectrometry of Small Samples with the Reflecting Microscope, "by R.C. Gore in Science (December 30, 1940): (C) 1940 by the American Association for the Advancement of Science Reprinted with permission.

This early research created sufficient interest that, in 1953, V. J. Coates (while employed by Perkin Elmer) developed a microscope for infrared analysis: the Perkin Elmer model 85, the first commercial infrared microspectrometer. This microscope was designed for use with a singlebeam scanning infrared monochrometer; while capable of producing spectra of small particles (see Fig. 2), it was not widely accepted.

From 1953 until 1978, infrared microspectroscopy remained a curiosity rather than a viable analytical technique. While early researchers demonstrated its principles, its feasibility, and its promise, the development of infrared microspectroscopy was halted until computers became more readily available and affordable. A quarter-century later, interest in infrared microspectroscopy revived when Coates introduced the Nanometrics model 20-IR. In 1978, Coates (now President of Nanometics, Inc.) developed a new infrared microspectrometer. He applied advances in microprocessor and detector technology to produce an infrared microspectrometer system that was easy to use, overcoming the limitations of the Perkin Elmer microscope. The computer digitized the spectral data, stored background and single-beam sample spectra, and computed good-quality ratioed spectra. The Nanospec 20IR demonstrated that infrared microspectroscopy was a practical and useful technique.

FT-IR spectrometers have several advantages for microspectroscopy: energy throughput, spectral resolution, high signal-to-noise, rapid data collection, and the ability to perform digital processing of acquired spectra. At the Inter/Micro '82 Meeting (in Chicago, IL), R. Z. Muggli reported the first successful adaptation of a microscope to a Fourier transform spectrometer: his attachment (in 1981) of a Perkin Elmer Model 85 microscope to a Digilab FTS15 spectrometer. With this system, Muggli demonstrated the practical advantages of FT-IR microspectroscopy. This system remained in operation at McCrone Associates for almost a decade. Muggli's success, along with a growing interest throughout the technical markeplace regarding the use of FT. IR microsampling in the semiconductor industry, stimulated sufficient interest for Digilab to contract with Spectra-Tech to design and manufacture a microscope exclusively for use with Digilab's FT-IR spectrometers. The result was Digilab's UMA-100, designed by D. W. Sting and R. G. Messerschmidt and introduced in 1983. At the Inter/Micro ' 84 Conference, K. Krishnan described Digilab's experience with FT-IR microscopy.

Another significant development was the work of Shearer and Cournoyer (at Kodak), which evolved into the Analect Instruments (a division of LaserPrecision Corp.) microscope. These researchers published a paper in the December 1977 issue of Analytical Chemistry entitled "Fourier Transform Infrared Analysis Below the One-Nanogram Level". This work was performed using only a beam condenser, with samples mounted over small pinhole apertures. Their experience convinced Shearer and Cournoyer that they should look for "a super beam condenser with viewing capabilities": a microscope. In 1979, they proposed an internal project at Kodak to build an infrared microscope. Unfortunately, this project's cost and complexity grew, interest waned, and the project was never completed. In 1982, Shearer became a consultant with Analect. In the Fall of 1983, Analect's M. Doyle approved Shearer's proposal to design a microscope for Analect's AQS-20 spectrometer. This resulted in the introduction (in 1984) of the FXA-515 microscope accessory; this was a transmission-only microscope. The Analect microscope was very well-accepted during the 1984-87 period.
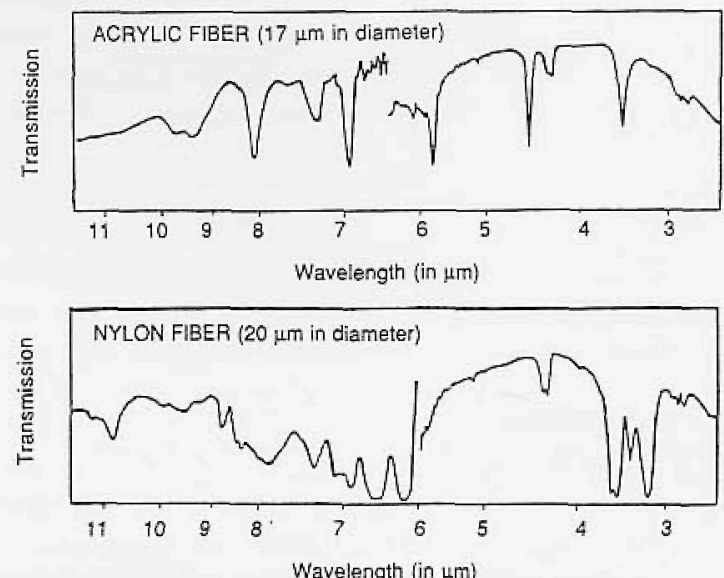

Figure 2: Infrared spectra of microscopic fibers from "Design and Performance of an Infrared Microscope Attachment," by V.J. Coates, A. Offner, and E.H. Siegler, Jr. in Journal of the Optical Society of America (Vol. 43, No. 11), p. 988

The first FT-IR microscope to combine the high-quality visual imaging of a research light microscope with the all-reflecting optics needed for infrared microspectroscopy was Spectra-Tech's IR-Plan ${ }^{\circ}$. Introduced in 1986, the IRPlan FT-IR microscope (developed by D. W. Sting, G. Ressler, and R. G. Messerschmidt) incorporated high-quality imaging standards with an improved Schwarzschild reflecting objective (designed by D. Schaefer), advanced optical design for higher spatial definition of a sample during infrared analysis, and remote-aperture beam splitting for improved reflected-infrared analysis. One 
major IR-Plan innovation was the introduction of dual confocal remote apertures to more precisely define a sample area by reducing the effects of diffraction on spectral measurements. With its advances in imaging and spectral performance, the IR-Plan microscope became widely accepted as the standard of comparison for all accessory microscopes. Prior to the development of the IR-Plan, infrared microscopes were primarily microsampling accessories for infrared spectroscopy, with little regard for the scientist's needs for high-quality visual imaging or photometic accuracy in infrared-microprobe (IRM) analysis.

Several versions of accessory microscopes have been commercially available from the major spectrometer manufacturers. In 1984, Bruker introduced its microscope accessory, designed by A. Simon. In 1987, Digilab redesigned its UMA 100 to improve performance, creating the UMA 300 , and also designed a lower-cost UMA 150 (in 1990) and a research microscope, the UMA 500 (in 1992). Perkin Elmer returned to the microscope accessory field in 1989 with a lower-cost microsampling accessory designed exclusively for its spectrometers. Mattson Instrument $\mathrm{Co}$. first introduced the Bach-Shearer microscope in 1987; unfortunately, this was not a competitive accessory. In 1992, the company again introduced an exclusive microscope product, the Quantum microsampling accessory (designed by $\mathrm{R}$. G. Messerschmidt). This lower-cost microsampling accessory employs conventional infrared beam-condenser optics with independent viewing optics. Harrick Scientific Corp. developed an in-compartment microsampling accessory, the IRS Nanosampler ${ }^{*}$, which provides both viewing and infrared spectral analysis. For the lower-cost market, Spectra-Tech developed two in-compartment microscopes, the Spectra-Scope ${ }^{\circ}$ (designed by D. W. Sting in 1985) and the E-Z Scope ${ }^{\circ}$ (designed in 1992 by P. Brierley and S. Vogel), as well as a series of accessory microscopes, including the Analytical IR-
Plan $^{\circ}$ (designed in 1988) by G. Ressler, S. Vogel, and C. Alexey.

The first totally-dedicated infrared microspectrometer system was introduced by Spectra-Tech at the Microbeam Analysis Society's annual meeting in 1989 (in Asheville, NC). This unique system, named $\mathbb{R}^{\mu S} \otimes$, integrates the microscope, the spectrometer, and the computer into a unified analytical instrument. Through integration, this IRM system (designed by G. Ressler, D. W. Sting, and J. A. Reffner) automates microscope operation and linkage to the spectrometer. The $\mathrm{IR}^{\mu S}$ development program was a joint project of SpectraTech and Nicolet Instrument Corp., with Nicolet currently marketing both the $\mathrm{IR}^{\mu \mathrm{S}}$ scanning-infrared-microprobe system and the Nic-Plan ${ }^{\Theta}$ accessory microscope for its FT-IR benches. The Nic-Plan is the $\mathrm{IR}^{\mu \mathrm{S}}$ microscope optical bench interfaced exclusively with Nicolet FT-IR spectrometers.

Present IRM designs allow either transmission or reflection spectra to be readily recorded. Their operation is direct and simple: While viewing a sample, its microstructure is studied, and areas are defined for infrared spectral measurement by image-plane masks. Confocal dual remote-image-plane masks minimize diffraction effects and provide the highest definition of a sample for infrared measurement.

Instrumentation development has moved in two directions: higher performance and lower cost. While these are divergent goals at present, in the long term, the cost of higher performance should be reduced, while the performance of lower-cost systems should improve. In addition, analytical capabilities have been extended to include internal-reflection and grazingincidence-reflection measurements on a microscopic scale. As each advance in the commercial development of instrumentation was made, the applications of the technology have increased. Today, there ar more than 3,000 infrared microscopes in use worldwide - and growth continues.

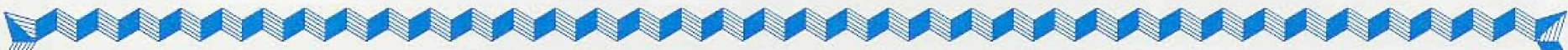 "Why look anywhere else?"}

Why does the Nicolet/Spectra-Tech team have more microscopes in the field than everyone else combined? Ask yourself these questions:

Would other FT-IR microscopes offer Redundant Aperturing for improved spatial resolution if they could beat our patents?

Would they offer ATR and grazing angle microsampling if they could?

\section{(You bet they would!)}

Full automation, the clearest and most flexible viewing optics and unequaled performance mean you get more work accomplished every day. So if you're seriously looking for an infrared microspectroscopy system, contact Nicolet/Spectra-Tech.

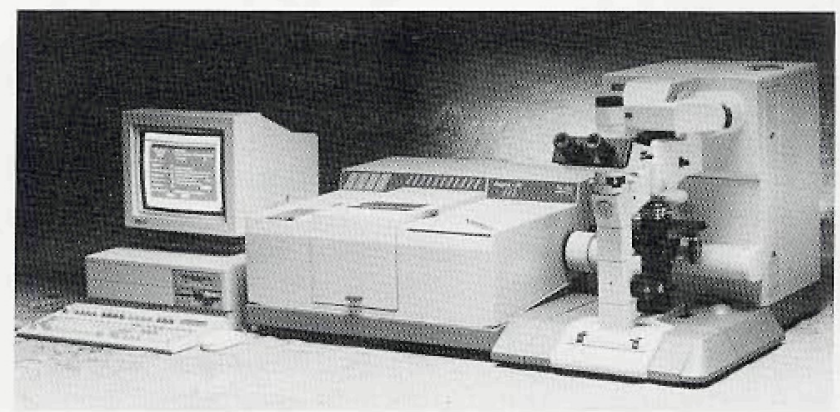

The complete line of Spectra-Tech products is offered only by Nicolet, backed by the most powerful and popular FT-IR spectrometers in the world. Call us Toll-free at 1-800-356-8088, or fax us at 608-273-5046, to receive more information.

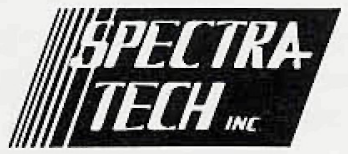

